\title{
Disease Progression and Mutation Pattern in a Large Cohort of LGMD R1/LGMD 2A Patients from India
}

Valakunja H. Ganaraja ${ }^{1, *}$ Kiran Polavarapu ${ }^{1,2, *}$ Mainak Bardhan ${ }^{1}$ Veeramani Preethish-Kumar ${ }^{1}$ Shingavi Leena ${ }^{1}$ Ram M. Anjanappa ${ }^{1,2}$ Seena Vengalii ${ }^{1}$ Saraswati Nashi ${ }^{1}$ Gautham Arunachal ${ }^{3}$ Swetha Gunasekaran ${ }^{3}$ Dhaarini Mohan ${ }^{1}$ Sanita Raju${ }^{1}$ Gopikrishnan Unnikrishnan ${ }^{1}$ Akshata Huddar ${ }^{1}$ Valasani Ravi-Kiran ${ }^{1}$ Priya T. Thomas ${ }^{4}$ Atchayaram Nalini ${ }^{1}$

1 Department of Neurology, National Institute of Mental Health and Neurosciences, Bengaluru, Karnataka, India

2 Division of Neurology, Department of Medicine, Children's Hospital of Eastern Ontario Research Institute, University of Ottawa, The Ottawa Hospital, Ottawa, Canada

${ }^{3}$ Department of Human Genetics, National Institute of Mental Health and Neurosciences, Bengaluru, Karnataka, India

${ }^{4}$ Department of Psychiatric Social Work, National Institute of Mental Health and Neurosciences, Bengaluru, Karnataka, India
Address for correspondence Atchayaram Nalini, DM, PhD, Department of Neurology, Neuroscience Faculty Center, National Institute of Mental Health and Neurosciences, Bengaluru 560029, Karnataka, India (e-mail: atchayaramnalini@yahoo.co.in).

Glob Med Genet 2022;9:34-41.

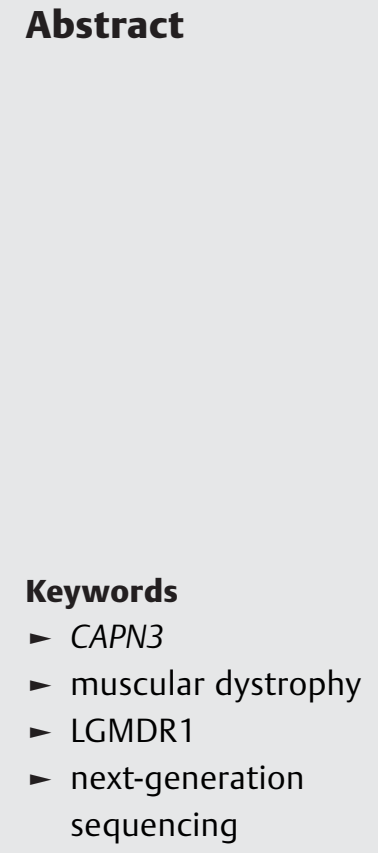

Calpainopathy is caused by mutations in the CAPN3. There is only one clinical and genetic study of CAPN3 from India and none from South India. A total of 72 (male[M]: female $[\mathrm{F}]=34: 38$ ) genetically confirmed probands from 72 independent families are included in this study. Consanguinity was present in $54.2 \%$. The mean age of onset and duration of symptoms are $13.5 \pm 6.4$ and $6.3 \pm 4.7$ years, respectively. Positive family history occurred in $23.3 \%$. The predominant initial symptoms were proximal lower limb weakness (52.1\%) and toe walking (20.5\%). At presentation, $97.2 \%$ had hip girdle weakness, $69.4 \%$ had scapular winging, and $58.3 \%$ had contractures. Follow-up was available in $76.4 \%$, and $92.7 \%$ were ambulant at a mean age of $23.7 \pm 7.6$ years and duration of 4.5 years, remaining $7.3 \%$ became wheelchair-bound at $25.5 \pm 5.7$ years of age (mean duration $=13.5 \pm 4.6$ ), $4.1 \%$ were aged more than 40 years (duration range $=5-20$ ). The majority remained ambulant 10 years after disease onset. Nextgeneration sequencing (NGS) detected 47 unique CAPN3 variants in 72 patients, out of which 19 are novel. Missense variants were most common occurring in $59.7 \%$ (homozygous $=29$; Compound heterozygous $=14$ ). In the remaining 29 patients (40.3\%), at least one suspected loss of function variant was present. Common recurrent variants were c.2051-1G $>T$ and c.2338G $>C$ in $9.7 \%, c .1343 G>A$, c.802-9G > A, and c.1319G $>A$ in $6.9 \%$ and c.1963delC in 5.5\% of population. Large

\footnotetext{
* These two authors contributed equally to this work.
}

received

July 7, 2021

accepted after revision

September 18, 2021

published online

November 9, 2021
DOI https://doi.org/ $10.1055 / \mathrm{s}-0041-1736567$. ISSN 2699-9404.

\section{(C) 2021. The Author(s).}

This is an open access article published by Thieme under the terms of the Creative Commons Attribution License, permitting unrestricted use, distribution, and reproduction so long as the original work is properly cited. (https://creativecommons.org/licenses/by/4.0/) Georg Thieme Verlag KG, Rüdigerstraße 14, 70469 Stuttgart, Germany 
deletions were observed in 4.2\%. Exon 10 mutations accounted for 12 patients (16.7\%). Our study highlights the efficiency of NGS technology in screening and molecular diagnosis of limb-girdle muscular dystrophy with recessive form (LGMDR1) patients in India.

\section{Introduction}

Calpainopathy caused by mutations in the CAPN3 gene (OMIM:114240) is one of the most common types of autosomal recessive limb-girdle muscular dystrophy (ARLGMD). The disease onset ranges from early childhood to adulthood and is typically characterized by progressive symmetrical weakness and wasting of proximal limb muscles and contractures. ${ }^{1}$ While the recessive form of LGMD (LGMDR1) is the most common one, the autosomal dominant form (LGMDD4) of calpainopathy has been reported in few families with adult onset and milder phenotypes. ${ }^{2,3}$ CAPN3 gene encodes a protease called calpain 3 and is located in the chromosome region 15q15.1-q21.1. ${ }^{4}$ CAPN3 is a $\mathrm{Ca}^{2+}$ dependent protein comprising of 821 amino acids. ${ }^{5,6}$ The gene consists of 24 exons and produces a skeletal muscle-specific homodimer cysteine protease belonging to the calpain superfamily which is involved in myofibrillogenesis and sarcomere remodeling. ${ }^{7-10}$ The prevalence of calpainopathy among all dominant and recessive forms of LGMD's ranges from $11 \%$ in the United States and Mexico, ${ }^{11-13}$ to 40 to $50 \%$ in Turkey, India, and Bulgaria, ${ }^{14-17}$ and even higher in Basque and Russia population. ${ }^{18-20}$

Although a few reports based on western blot confirmation of calpainopathy are published on Indian patients, ${ }^{21,22}$ only two studies describe the mutation pattern in the Indian community with calpainopathy and none on the disease progression. ${ }^{23,24}$ Additionally, a few international studies from Italy and France reported on the natural history/disease progression. ${ }^{25-27}$

In the current study, we elaborated on the clinical presentation, disease progression, and mutation pattern of a large cohort of genetically confirmed LGMDR1 patients evaluated at a single quaternary referral center for neurological disorders in India. Since we will be discussing only the recessive form, the term "LGMDR1" will be used synonymously with "calpainopathy."

\section{Materials and Methods}

Institutional ethics committee approval for the study was obtained (NIMHANS/IEC/2020-21). This is a retrospective analysis with a description of the clinical phenotype and mutation pattern in 72 patients of genetically confirmed LGMDR1 patients. They belonged to a cohort of 345 ARLGMD patients evaluated between 2016 and 2020 at the multidisciplinary neuromuscular disorders clinic. After detailed history taking and neurological examination of all patients, relevant demographic and clinical data were entered in SPSS (Statistical Package for the Social Sciences) for further statistical analysis. It is commonly used for analysis of complex statistical data. The muscle strength was evaluated by the modified Medical Research Council (MRC) grading scale ${ }^{28}$ which is an objective method used clinically to assess individual muscle weakness. After obtaining written informed consent, patients underwent genetic testing by next-generation sequencing (NGS) including clinical exome or whole exome sequencing. The libraries were sequenced on the Illumina sequencing platform, followed by bioinformatic analysis customized for calling single nucleotide variants (SNVs), small insertion/deletions (INDELs), as well as larger copy number variants (CNVs; exon deletions and duplications). CAPN3 variant annotation was done based on GenBank transcript: NM_000070.3. We used the free version of VarSome online tool (https://www.varsome.com/) and manual application of American College of Medical Genetics and Genomics (ACMG) criteria $(2015)^{29}$ for classification of SNVs and INDELs. CNVs when identified were automatically considered as causing loss of function and classified as pathogenic. Multiplex ligation-dependent probe amplification (MLPA) was also performed in two patients with large multiexon deletions identified by NGS.

We checked available data in Clinvar, Human Gene Mutation Database (HGMD), Leiden variant database (https:// databases.lovd.nl/shared/genes/CAPN3), and literature for identification of reported variants. Demographic and phenotype data of patients were entered in SPSS Version 23 for statistical analysis. To study the disease progression with respect to time to loss of independent ambulation and time to wheel chair and bed bound state, all the details were obtained from the medical records. A simple questionnaire with information for functional and physical disability was posted to all genetically confirmed LGMDR1 patients to collect their latest disability status.

\section{Statistics}

Data were analyzed in SPSS version 23 using descriptive statistics, such as mean and standard deviation for continuous variables. Frequency percentage was used for categorical variables. Comparison between two genotype groups was performed by independent sample $t$-test. The significance level ( $p$-value) was fixed at 0.05 .

\section{Results}

Among the 345 genetically confirmed ARLGMD patients, we identified 72 LGMDR1 (20.9\%) individuals from 72 independent families. The mean age at onset was $13.5 \pm 6.4$ (range: $1-35$ ) years, and the mean duration was $6.3 \pm 4.7$ (range: $0.4-20$ ) years. There were 34 (47.2\%) males. Patients from the 
southern states of India formed $75.4 \%$ of the study cohort, followed by $17.8 \%$ from the Eastern states, while the remaining $(6.9 \%)$ were from the West and Northern parts. Consanguinity was reported in 39 (54.2\%) patients, and a family history of similar illness was present in 17 (23.3\%). About 29 (40.3\%) patients had onset before 12 years of age, while 35 (48.6\%) had onset between 12 and 20 years of age, and only 8 (11.1\%) had onset after 20 years. The initial symptoms were progressive proximal lower limb weakness (52.1\%), toe walking (20.5\%), and difficulty running fast (9.6\%), while a small proportion (5.5\%) had a combination of these at the onset. The clinical features are summarized in - Table 1.

At presentation, 70 (97.2\%) patients had difficulty in rising from the floor and climbing stairs from mean ages of $14.5 \pm 6.1$ and $14.9 \pm 5.8$ years, respectively. Recurrent falls were reported by 36 (50\%) patients from a mean age of $15.6 \pm 6.0$ years. About $48(66.6 \%)$ patients presented with proximal upper limb weakness from $16.7 \pm 5.7$ years of age, and a small proportion of $6(8.3 \%)$ patients had features of distal upper limb weakness with later onset at the age of $17.7 \pm 4.8$ years. About 41 (56.9\%) patients had observed that they had difficulty in running like others from a mean age of $14.7 \pm 6.8$ years. Other presentations were muscle pain and exercise intolerance in $12(16.6 \%)$ and 9 (12.5\%) patients, respectively. Two (2.7\%) were in wheelchair/bedbound state at 18 and 26 years of age with 11 and 14 years into their illness, respectively. None of the patients had symptoms to suggest cranial muscle involvement.

On examination, approximately 28 (38.8\%) had prominent lumbar lordosis, 24 (33.3\%) had mild calf hypertrophy, and $13(18.1 \%)$ patients had prominent atrophy of calves and arms. Scapular winging was noticed in 50 (69.4\%) and prominent contractures in 42 (58.3\%) patients, predominantly at the ankles and knees. Around 15 (20.8\%) patients had mild facial weakness. The muscle strength was severely reduced in the girdle muscles (-Fig. 1). Preferential involvement of the muscles was recorded: pectoralis and biceps muscles were more severely involved than deltoids and triceps. Iliopsoas, hip adductors, and gastrocnemius were more affected than the glutei and anterior leg muscles. The pattern of muscle involvement is summarized in -Table 2. Distal muscles were preserved in the majority of patients (-Fig. 1). All individuals had a waddling gait associated with prominent toe walking in 29 (40.3\%). Tendon reflexes were generally hypoactive but ankle jerk was preserved in 52 (72.2\%) patients.

\section{Genetic Findings}

Among the 72 genetically confirmed LGMDR1 patients, 57 had homozygous and the remaining 15 had compound heterozygous mutations in the CAPN3 gene. Among the compound heterozygous cases, all except two had missense variant in one allele with suspected loss of function variant like frameshift causing INDEL or splice affecting intronic substitution in the opposite allele. Two compound heterozygous patients had biallelic INDELs and missense variants, respectively (Pt_20 and Pt_28). Out of 57 homozygous cases, 29 had missense variants, 28 had suspected loss of function variants (splice
Table 1 Clinical profile of 72 patients of calpainopathy

\begin{tabular}{|c|c|c|}
\hline Parameter & $\begin{array}{l}\text { Total number } \\
(n=72)\end{array}$ & Percentage \\
\hline Duration of illness (y) & $6.3 \pm 4.7$ & \\
\hline Male:female & $34: 38$ & \\
\hline Consanguinity & 39 & 54.2 \\
\hline Family history & 17 & 23.3 \\
\hline \multicolumn{3}{|l|}{ Initial symptom } \\
\hline Age of onset (y) & $13.5 \pm 6.4$ & \\
\hline $\begin{array}{l}\text { Proximal lower limb } \\
\text { weakness }\end{array}$ & 38 & 52.1 \\
\hline Toe walking & 15 & 20.5 \\
\hline $\begin{array}{l}\text { Difficulty in running } \\
\text { fast }\end{array}$ & 7 & 9.6 \\
\hline $\begin{array}{l}\text { Combination of } \\
\text { symptoms }\end{array}$ & 4 & 5.5 \\
\hline Repeated falls & 4 & 5.5 \\
\hline Muscle pain & 2 & 2.8 \\
\hline Calf swelling & 1 & 1.4 \\
\hline $\begin{array}{l}\text { Delayed motor } \\
\text { milestones }\end{array}$ & 1 & 1.4 \\
\hline \multicolumn{3}{|c|}{ Symptoms at presentation } \\
\hline $\begin{array}{l}\text { difficulty in climbing } \\
\text { stairs }\end{array}$ & 70 & 97.2 \\
\hline $\begin{array}{l}\text { Difficulty in getting } \\
\text { up }\end{array}$ & 70 & 97.2 \\
\hline $\begin{array}{l}\text { Proximal upper } \\
\text { limb weakness }\end{array}$ & 48 & 66.6 \\
\hline Running difficulty & 41 & 56.9 \\
\hline Recurrent falls & 36 & 50 \\
\hline Muscle pain & 12 & 16.6 \\
\hline Exercise intolerance & 9 & 12.5 \\
\hline $\begin{array}{l}\text { Distal upper limb } \\
\text { weakness }\end{array}$ & 6 & 8.3 \\
\hline $\begin{array}{l}\text { Distal lower limb } \\
\text { weakness }\end{array}$ & 2 & 2.7 \\
\hline \multicolumn{3}{|l|}{ Examination } \\
\hline Scapular winging & 50 & 69.4 \\
\hline Contractures & 42 & 58.3 \\
\hline Toe walking & 29 & 40.2 \\
\hline Lumbar lordosis & 28 & 38.8 \\
\hline Calf hypertrophy & 24 & 33.3 \\
\hline
\end{tabular}

affecting intronic variants $=13$; INDELs causing frameshift $=10$; multiexonic deletions $=3$; inframe 3 bp deletion $=1$; and substitution resulting in nonsense codon $=1$ ). In total, 47 unique disease-causing CAPN3 variants were identified in this study, out of which 19 are novel mutations (-Fig. 2; -Supplementary Table S1, available in online version only). 


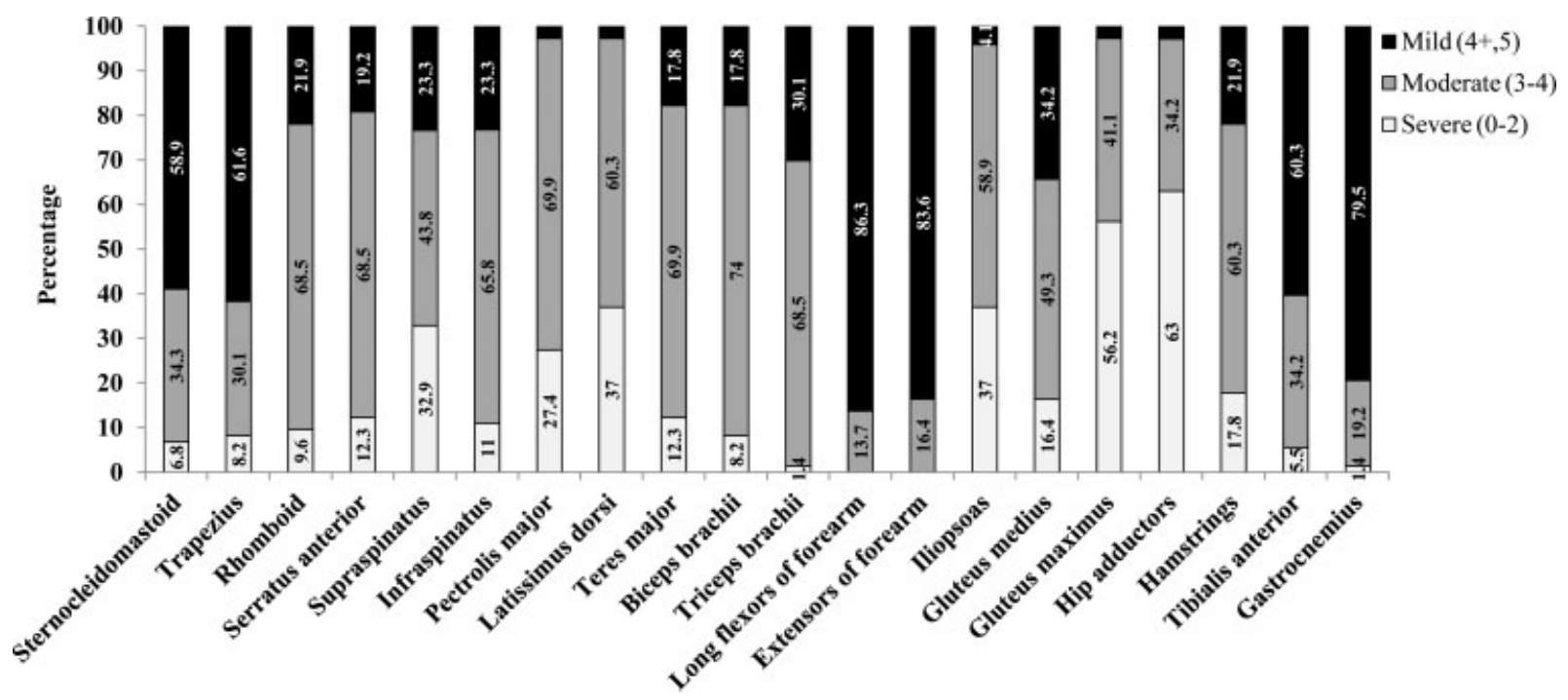

Fig. 1 Severity of various muscle weakness among 72 patients with "calpainopathy."

While disease causing variants are scattered across the CAPN3 gene with no specific hotspot regions, we identified few recurrent variants in Indian patients (-Table 4). The commonest mutation identified are c.2051-1G $>\mathrm{T}$ and c.2338G $>C$ in seven (9.7\%) patients each. Other mutation clusters were c.802-9G $>$ A, c.1319G $>A$, and c.1343G $>$ A occurring in five $(6.9 \%)$ patients each, while c.1963delC was identified in four (5.5\%) patients. Exon 10 was most frequently involved in our patients with 12 mutations (16.7\%). No muta-

Table 2 Muscle selectivity pattern in calpainopathy patients

\begin{tabular}{|c|c|c|}
\hline Selectivity pattern & Number & Percentage \\
\hline \multicolumn{3}{|l|}{ Upper limbs } \\
\hline Deltoid $>$ pectoralis & 8 & 10.9 \\
\hline Deltoid $=$ pectoralis & 25 & 35.6 \\
\hline Deltoid $<$ pectoralis & 39 & 53.4 \\
\hline \multicolumn{3}{|l|}{ Arm } \\
\hline Biceps $>$ triceps & 36 & 50.6 \\
\hline Biceps $=$ triceps & 29 & 39.7 \\
\hline Biceps $<$ triceps & 7 & 9.5 \\
\hline \multicolumn{3}{|l|}{ Lower limbs } \\
\hline Iliopsoas > gluteus maximus & 15 & 20.5 \\
\hline Iliopsoas = gluteus maximus & 26 & 35.6 \\
\hline Iliopsoas < gluteus maximus & 31 & 43.8 \\
\hline \multicolumn{3}{|l|}{ Thigh } \\
\hline Adductors $>$ abductors & 49 & 67.1 \\
\hline Adductors $=$ abductors & 19 & 27.4 \\
\hline Adductors $<$ abductors & 4 & 5.5 \\
\hline \multicolumn{3}{|l|}{ Leg } \\
\hline Gastrocnemius $>$ tib anterior & 4 & 5.5 \\
\hline Gastrocnemius $=$ tib anterior & 51 & 69.9 \\
\hline Gastrocnemius $<$ tib anterior & 17 & 24.7 \\
\hline
\end{tabular}

tions were observed in exons 2, 3, 7, and 12. Large deletions were observed between exons 17 to 24 in three unrelated patients by NGS. We confirmed the deletion in two of the three patients with additional deletion/duplication analysis by MLPA. The family members of 31 patients were screened by segregation analysis and at least one member was found to be having a heterozygous state, and six members with similar illness were detected to have homozygous status (-Supplementary Table S2, available in online version only).

\section{Genotypic Patterns and Disease Progression}

Clinical follow-up details were available in 55 (76.4\%) patients with a mean duration of $4.5 \pm 4.3$ years. About $92.7 \%$ of them were ambulant at a mean age of $23.7 \pm 7.6$ years, while the remaining $7.3 \%$ (four patients) had become wheelchair bound at a mean age of $25.5 \pm 5.7$ years, after 13.5 $(13.5 \pm 4.6)$ years into the illness. On subgroup analysis among patients with different mutations, those with missense mutations had later age of presentation and slow disease progression than those with other mutations (-Table 3). Among patients in wheelchair-bound state, only one had missense mutation (Pt_64). Three patients (4.1\%) were available for follow-up with age more than 40 years and disease duration ranging from 5 to 20 years (Pt_26, Pt_36, and Pt_39); all were ambulant with assistance of which two were having missense mutations. The oldest patient with follow-up in our study cohort is 43 years, with 20 years of illness duration (Pt_26). One patient (Pt_17) with splice affecting mutation (c.802-9G $>$ A) had sudden death at 21 years of age after 7 years into the illness and was reported to be due to cardiac arrest; however, medical records are not available. None of the patients had features suggestive of respiratory distress during the period of clinical follow-up.

\section{Discussion}

In the current study, we present the results of clinical findings, pattern of disease progression, and genetic mutation profile of 


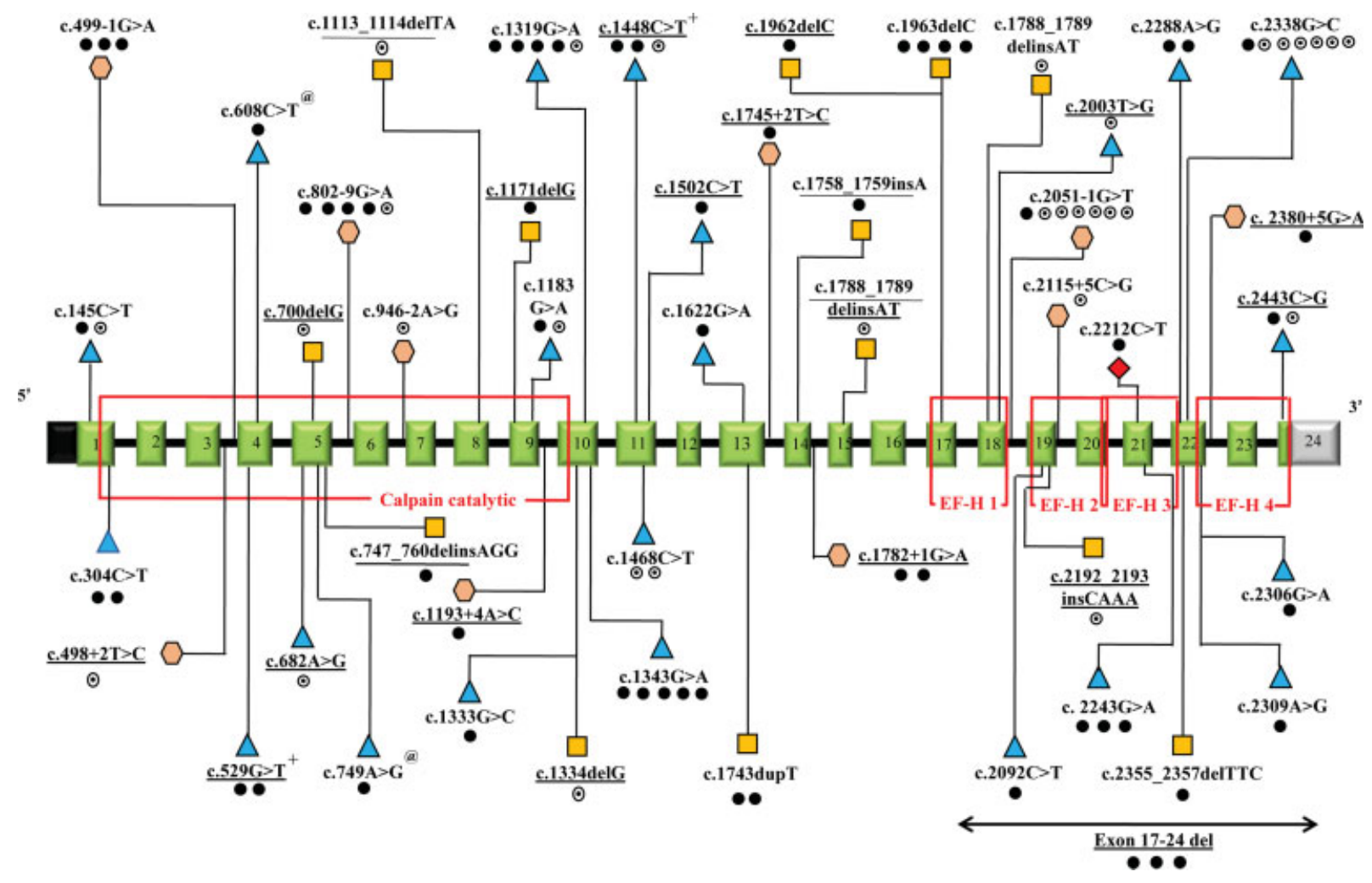

Fig. 2 Schematic representation of the variations identified in our study in CAPN3 gene with corresponding exons and protein domains. The 24 exons of CAPN3 (NM_000070.3) are represented as boxes with respective exonic numbers with noncoding regions shaded in black and gray at the ends. The domain information is based on UniProtKB - P20807 (CAN3_HUMAN) taken from Uniprot database (https://www.uniprot.org/). The novel variants are underlined. The dark dots represent homozygous variations, unfilled dots represent heterozygous variations, semifilled dot represent compound heterozygous variations. Triangles represent missense, squares represent frameshift, rhombus represent nonsense and hexagon represent intronic variations. ; @, +, - Individuals carry two homozygous variations in CAPN3. The size of exons /introns is not represented at scale. Calpain catalytic domain and EF-hand domains 1-4 are shown in red.

Table 3 Genotypic correlation among calpainopathy patients with clinical follow-up $(n=55)$

\begin{tabular}{|l|l|l|l|}
\hline Parameters & $\begin{array}{l}\text { Group 1: } \boldsymbol{n = 2 4} \\
\text { (missense mutation) }\end{array}$ & $\begin{array}{l}\text { Group 2: } \boldsymbol{n = 3 1} \\
\text { (other mutations) }\end{array}$ & $p$-Value \\
\hline Age of onset (y) & $16.4 \pm 7.0$ & $11.7 \pm 5.66$ & 0.07 \\
\hline Duration (y) & $6.4 \pm 4.4$ & $6.4 \pm 5.3$ & 0.9 \\
\hline Age at presentation (y) & $22.8 \pm 7.1$ & $18.13 \pm 7.0$ & 0.02 \\
\hline Male:female & $12: 12$ & $12: 19$ & NA \\
\hline Consanguinity (yes:no) & $14: 10$ & $17: 14$ & NA \\
\hline Presence of family history & 8 & 5 & NA \\
\hline Mean age of follow-up (y) & $25.4 \pm 7.5$ & $22.2 \pm 8.3$ & 0.14 \\
\hline Wheelchair bound status during follow-up & 1 & 3 & NA \\
\hline
\end{tabular}

Abbreviation: NA, not applicable.

a large number of LGMDR1 patients from India. The majority of them belong to southern India and demonstrated varied mutation patterns. In an earlier study from India, LGMDR1 is reported to be the commonest form of ARLGMD; however, there is no clear data about the exact prevalence and subtypes of genetically confirmed patients. ${ }^{14}$ In our study, LGMDR1 constituted $21.2 \%$ of all genetically confirmed ARLGMD patients.
The age of onset of muscle weakness in the present study is in concordance with previous studies ${ }^{20,22}$; however, a later age of onset has been noted in a study from Japan. ${ }^{30}$ Both genders were equally affected in our study, similar to the findings in other large series. ${ }^{31,32}$ However, in one study from India with 75 patients, male predominance was noted. ${ }^{14}$

Nearly half of our patients had proximal weakness of lower limbs as the initial presentation and almost $20 \%$ had 
Table 4 Recurrent CAPN3 variants in our cohort

\begin{tabular}{|c|c|c|c|c|}
\hline Variants & Number of patients & Patient ID & Location & Zygosity \\
\hline c. $2051-1 G>T$ & $7(9.7 \%)$ & Pt_52 to 58 & Intron18 & Heterozygous \\
\hline c.2338G > C(p.Asp780His) & $7(9.7 \%)$ & Pt_52 to 58 & Exon 22 & Heterozygous \\
\hline c.802-9G >A & $5(6.9 \%)$ & Pt_15 to Pt_19 & Intron 5 & Homozygous \\
\hline c.1319G > A(p.Arg440Gln) & $5(6.9 \%)$ & Pt_24 to Pt_28 & Exon 10 & Homozygous \\
\hline c.1343G > A(p.Arg448His) & $5(6.9 \%)$ & Pt_30 to Pt_34 & Exon 10 & Homozygous \\
\hline c.1963delC & $4(5.5 \%)$ & Pt_45 to 48 & Exon 17 & Homozygous \\
\hline c.145C > T(p.Arg49Cys) & $2(2.7 \%)$ & Pt_1, Pt_2 & Exon 1 & Homozygous \\
\hline c.304C > T(p.Pro102Ser) & $2(2.7 \%)$ & Pt_3, Pt_4 & Exon 1 & Homozygous \\
\hline c.529G > T(p.Val177Phe $)^{a}$ & $2(2.7 \%)$ & Pt_10, Pt_11 & Exon 4 & Homozygous \\
\hline c.1448C > T(p.Ala483Vala ${ }^{\mathrm{a}}$ & $2(2.7 \%)$ & Pt_10, Pt_11 & Exon 11 & Homozygous \\
\hline c.1743dupT(p.Glu582T) & $2(2.7 \%)$ & Pt_38, Pt_39 & Exon 13 & Homozygous \\
\hline c. $1782+1 G>A^{a}$ & $2(2.7 \%)$ & Pt_42, Pt_43 & Exon 17 & Homozygous \\
\hline
\end{tabular}

${ }^{\mathrm{a}}$ Novel variants.

tip-toe walking. Scapular winging was noted in $69 \%$ of patients. The commonest presentation described in other studies are similar with difficulty in running, tip-toe walking, and scapular winging along with proximal lower limb weakness. ${ }^{25,32,33}$ In a study by Peric et al, from Serbia, up to $74 \%$ of their patients had features of proximal lower limb weakness as the initial presentation, and scapular winging was noticed in more than two-thirds of their patients. ${ }^{34}$

The pectorals, biceps brachii, and gluteus maximus were the weakest muscles. This pattern of selective involvement is also reported in a previous study. ${ }^{35}$ The symmetrical and preferential muscle involvement also helps in identifying the clinical phenotype which could aid in precise genetic testing. ${ }^{36,37}$

In the course of disease progression, upper limb weakness was noted in about two-thirds of patients by 3 years of symptom onset and had limb-girdle weakness as was seen in our previous study. ${ }^{22}$ However, in one study from north India, only $16 \%$ of them had this feature, and this could be due to the varied time of evaluation after disease onset in these patients. ${ }^{14}$

The mean duration of follow-up and mean age at last follow-up were 4.5 and 23.7 years, respectively. The majority of our patients continued to be ambulant at a mean of 10 years after the onset of symptoms suggesting a relatively benign course of the illness. In one of the largest studies by Sáenz et al, among 238 LGMDR1 patients, the mean age at becoming wheel chair dependent was 32.2 years, approximately 18 years after the mean age of disease onset. ${ }^{32}$ In a study from Italy, Angelini et al have documented loss of ambulation from 10 to 30 years after disease onset, ${ }^{25}$ similar to the study from the United Kingdom, where loss of ambulation occurred at 35.2 years, that is, approximately 22.6 years after onset of symptoms. ${ }^{38}$ Similar observations were noted in other European reports. ${ }^{27,32}$ Hence, further longterm follow-up of our patients is necessary for assessing the future status of disease progression. In one of our patients with sudden death at 21 years of age, it was suspected to be due to myocardial infarction. In the previous studies, though respiratory insufficiency is frequently observed in calpainopathy patients, a few cases of cardiac involvement are also reported. ${ }^{39}$

Mutation analysis in our cohort has shown 47 different mutations, and the spectrum is distributed throughout the length of the CAPN3 gene (-Fig. 2). The majority of mutations are of missense type. Even previous studies have described 34 to $60 \%$ of mutations of missense type, and exons 10 and 21 were more susceptible than exons $2,3,7$, and $12 . .^{24,33}$

The mutational changes in exon 10 , that is, c.1343G $>A$, and c.1319G $>A$, leading to missense variation and amino acid substitution occurred in $13.8 \%$ of our population. This accumulation and gene pooling in our study cohort could be contributed by consanguinity which is more prevalent in southern India. Overall, 550 delA is the commonest among the Caucasian population with founder effect being demonstrated in Mediterranean and southern European region. ${ }^{40}$ However, this mutation was not found in our current Indian cohort. Founder mutations seen in the Brazilian population, R110X, and 2362-2363EG4TCATCT was also not identified in our study. ${ }^{41}$ Nevertheless, one founder variant (c.2306G > A) in exon 22, previously described in Amish community of northern Indiana of the United States was detected in one of our patients. ${ }^{4}$ Another variant previously described in La reunion island of Indian ocean (c.946-2A $>\mathrm{G}$ ) was also detected in our series. ${ }^{35}$

Seven mutations seen in previously described mutational "hotspot" of exon 21, constituted only approximately $6.9 \%$ in our study population. ${ }^{41}$ As the types of genetic mutations are known to vary from region to region and with ethnic background, the distribution of CAPN3 mutations in this study did not show any clear hot spots except for mutation accumulation in exon 10 in 12 cases. We also did not observe any mutations in exons $2,3,7$, and 12 . This wide range of mutations observed in our study could also be due to heterogeneous population of patients with a wide range of 
ethnicity. In another study from a north Indian center, exon 16 was the commonest mutation site, and approximately $75.6 \%$ of mutation groups were located around nine exons. ${ }^{24}$ This further strengthens the wide variation of genetic mutation from various regions within India.

There were two patients in the current cohort (Pt_10 and Pt_11) carrying two homozygous variations in CAPN3 at exon4/11 c.529G > T/c.1448C > T. Both had onset of symptoms at 15 and 18 years, respectively, with features of proximal lower limb weakness with one (Pt_11) having muscle pain as additional symptom. Though both were from different families, they belonged to a single community and during the follow-up duration of 6 years each, both were still ambulant. Both these novel mutations, c.529G $>$ T/c.1448C $>$ T in these patients were likely pathogenic based on ACMG classification suggesting their role in the clinical phenotype. Large exonic deletions are uncommon in CAPN3 but deletions from exons 2 to 8 have been described before. ${ }^{17}$ In our study, large deletions were observed in three unrelated patients. Interestingly, all belong to the Patel community which is believed to originate and be more prevalent in Gujarat. The Agarwal founder mutations: c.2051-1G $>$ T and c.2338G $>$ C were the most recurrent ones in our cohort occurring in total eight patients (compound heterozygous: 6 and homozygous: 2), all belonging to the Agarwal community as described before ${ }^{23}$ (-Table 4; - Supplementary Table S1, available in online version only). There was no direct correlation observed between the type of genetic mutation and course of disease progression, except those with missense mutation had a more benign course of disease progression than those with nonmissense mutations. However, observation about intrafamilial mutation pattern and disease progression could not be done.

The current study is one of the largest series describing the clinical phenotype, disease progression, and mutation patterns in genetically confirmed LGMDR1 and the first from south India. This study also shows the segregation of CAPN3 mutation in first-order relatives of the family (-Supplementary Table S2, available in online version only). This study also highlights mutation clusters around few exons, aiding in the diagnosis in limited settings.

\section{Limitations}

Major limitations of this study are that although it has identified genetic mutation clusters, a still larger sample size from each community needs to be screened for these mutations to look for founder effect in this region. Studying intrafamilial genetic variability with phenotypic assessment will further help in understanding this disease pattern. No longitudinal follow-up was performed to investigate the disease progression and natural history.

\section{Conclusion}

Hereby, we describe the genetic mutation pattern of CAPN3 gene and disease progression in Indian, predominantly south Indian patients of LGMDR1. Most of the patients develop symptoms in the second decade with proximal lower limb weakness. The predominant findings include abnormal gait with scapula winging. The majority of the patients remained ambulant even after 10 years of symptoms onset, suggesting a benign course of illness. There were 47 different mutations noted in these patients with 19 novel variants. These novel variants identified in CAPN3 expand the genotype-phenotype correlation associated with LGMDR1 in Indian population. Our study brings into light the usefulness of NGS for identifying novel variants in LGMDR1 and can be used as a stand-alone molecular diagnostic screening tool for all LGMD-related genes. However, additional confirmation might be required for larger deletions/duplications and complex rearrangements.

\section{Data Availability Statement}

The data that support the findings of this study are available from the corresponding author upon reasonable request.

\section{Funding}

None.

\section{Conflict of Interest}

None declared.

\section{Acknowledgments}

The authors thank the patients and their families for participating in this study.

\section{References}

1 Dinçer P, Leturcq F, Richard I, et al. A biochemical, genetic, and clinical survey of autosomal recessive limb girdle muscular dystrophies in Turkey. Ann Neurol 1997;42(02):222-229

2 Martinez-Thompson JM, Niu Z, Tracy JA, et al. Autosomal dominant calpainopathy due to heterozygous CAPN3 C.643_663del21. Muscle Nerve 2018;57(04):679-683

3 Vissing J, Barresi R, Witting N, et al. A heterozygous 21-bp deletion in CAPN3 causes dominantly inherited limb girdle muscular dystrophy. Brain 2016;139(Pt 8):2154-2163

4 Richard I, Broux O, Allamand V, et al. Mutations in the proteolytic enzyme calpain 3 cause limb-girdle muscular dystrophy type $2 \mathrm{~A}$. Cell 1995;81(01):27-40

5 Campbell RL, Davies PL. Structure-function relationships in calpains. Biochem J 2012;447(03):335-351

6 Sorimachi H, Hata S, Ono Y. Calpain chronicle-an enzyme family under multidisciplinary characterization. Proc Jpn Acad, Ser B, Phys Biol Sci 2011;87(06):287-327

7 Beckmann JS, Richard I, Hillaire D, et al. A gene for limb-girdle muscular dystrophy maps to chromosome 15 by linkage. C R Acad Sci III 1991;312(04):141-148

8 Sorimachi H, Imajoh-Ohmi S, Emori Y, et al. Molecular cloning of a novel mammalian calcium-dependent protease distinct from both $\mathrm{m}$ - and mu-types. Specific expression of the mRNA in skeletal muscle. J Biol Chem 1989;264(33):20106-20111

9 Kramerova I, Kudryashova E, Tidball JG, Spencer MJ. Null mutation of calpain 3 (p94) in mice causes abnormal sarcomere formation in vivo and in vitro. Hum Mol Genet 2004;13(13):1373-1388

10 Partha SK, Ravulapalli R, Allingham JS, Campbell RL, Davies PL. Crystal structure of calpain-3 penta-EF-hand (PEF) domain - a homodimerized PEF family member with calcium bound at the fifth EF-hand. FEBS J 2014;281(14):3138-3149 
11 Chou FL, Angelini C, Daentl D, et al. Calpain III mutation analysis of a heterogeneous limb-girdle muscular dystrophy population. Neurology 1999;52(05):1015-1020

12 Gómez-Díaz B, Rosas-Vargas H, Roque-Ramírez B, et al. Immunodetection analysis of muscular dystrophies in Mexico. Muscle Nerve 2012;45(03):338-345

13 Moore SA, Shilling CJ, Westra S, et al. Limb-girdle muscular dystrophy in the United States. J Neuropathol Exp Neurol 2006; 65(10):995-1003

14 Pathak P, Sharma MC, Sarkar C, et al. Limb girdle muscular dystrophy type $2 \mathrm{~A}$ in India: a study based on semi-quantitative protein analysis, with clinical and histopathological correlation. Neurol India 2010;58(04):549-554

15 Todorova A, Georgieva B, Tournev I, et al. A large deletion and novel point mutations in the calpain 3 gene (CAPN3) in Bulgarian LGMD2A patients. Neurogenetics 2007;8(03):225-229

16 Balci B, Aurino S, Haliloglu G, et al. Calpain-3 mutations in Turkey. Eur J Pediatr 2006;165(05):293-298

17 Richard I, Brenguier L, Dinçer P, et al. Multiple independent molecular etiology for limb-girdle muscular dystrophy type $2 \mathrm{~A}$ patients from various geographical origins. Am J Hum Genet 1997;60(05):1128-1138

18 Dadali EL, Shagina OA, Ryzhkova OP, Rudenskaia GE, Fedotov VP, Poliakov AV. [Clinical-genetic characteristics of limb girdle-muscular dystrophy type 2A]. Zh Nevrol Psikhiatr Im S S Korsakova 2010;110(04):79-83

19 Pogoda TV, Krakhmaleva IN, Lipatova NA, Shakhovskaya NI, Shishkin SS, Limborska SA. High incidence of 550delA mutation of CAPN3 in LGMD2 patients from Russia. Hum Mutat 2000;15(03):295

20 Urtasun M, Sáenz A, Roudaut C, et al. Limb-girdle muscular dystrophy in Guipúzcoa (Basque Country, Spain). Brain 1998; 121(pt. 9):1735-1747

21 Renjini R, Gayathri N, Nalini A, Srinivas Bharath MM. Analysis of calpain-3 protein in muscle biopsies of different muscular dystrophies from India. Indian J Med Res 2012;135(06):878-886

22 Nalini A, Polavarapu K, Sunitha B, et al. A prospective study on the immunophenotypic characterization of limb girdle muscular dystrophies 2 in India. Neurol India 2015;63(04):548-560

23 Khadilkar SV, Chaudhari CR, Dastur RS, Gaitonde PS, Yadav JG. Limb-girdle muscular dystrophy in the Agarwals: utility of founder mutations in CAPN3 gene. Ann Indian Acad Neurol 2016;19(01):108-111

24 Pathak P, Sharma MC, Jha P, et al. Mutational Spectrum of CAPN3 with genotype-phenotype correlations in limb girdle muscular dystrophy type 2A/R1 (LGMD2A/LGMDR1) patients in India. J Neuromuscul Dis 2021;8(01):125-136

25 Angelini C, Nardetto L, Borsato C, et al. The clinical course of calpainopathy (LGMD2A) and dysferlinopathy (LGMD2B). Neurol Res 2010;32(01):41-46

26 Richard I, Hogrel JY, Stockholm D, et al; Calpainopathy Study Group. Natural history of LGMD2A for delineating outcome measures in clinical trials. Ann Clin Transl Neurol 2016;3(04): 248-265

27 Fanin M, Nardetto L, Nascimbeni AC, et al. Correlations between clinical severity, genotype and muscle pathology in limb girdle muscular dystrophy type 2A. J Med Genet 2007;44(10):609-614

28 Vanhoutte EK, Faber CG, van Nes SI, et al; PeriNomS Study Group. Modifying the Medical Research Council grading system through Rasch analyses. Brain 2012;135(Pt 5):1639-1649

29 Richards S, Aziz N, Bale S, et al; ACMG Laboratory Quality Assurance Committee. Standards and guidelines for the interpretation of sequence variants: a joint consensus recommendation of the American College of Medical Genetics and Genomics and the Association for Molecular Pathology. Genet Med 2015;17(05): 405-424

30 Chae J, Minami N, Jin Y, et al. Calpain 3 gene mutations: genetic and clinico-pathologic findings in limb-girdle muscular dystrophy. Neuromuscul Disord 2001;11(6,7):547-555

31 Magri F, Nigro V, Angelini C, et al. The italian limb girdle muscular dystrophy registry: relative frequency, clinical features, and differential diagnosis. Muscle Nerve 2017;55(01):55-68

32 Sáenz A, Leturcq F, Cobo AM, et al. LGMD2A: genotype-phenotype correlations based on a large mutational survey on the calpain 3 gene. Brain 2005;128(pt. 4):732-742

33 Fanin M, Angelini C. Protein and genetic diagnosis of limb girdle muscular dystrophy type 2A: the yield and the pitfalls. Muscle Nerve 2015;52(02):163-173

34 Peric S, Stevanovic J, Johnson K, et al. Phenotypic and genetic spectrum of patients with limb-girdle muscular dystrophy type 2A from Serbia. Acta Myol 2019;38(03):163-171

35 Fardeau M, Hillaire D, Mignard C, et al. Juvenile limb-girdle muscular dystrophy. Clinical, histopathological and genetic data from a small community living in the Reunion Island. Brain 1996; 119(pt. 1):295-308

36 Fanin M, Nascimbeni AC, Angelini C. Muscle atrophy in Limb Girdle Muscular Dystrophy 2A: a morphometric and molecular study. Neuropathol Appl Neurobiol 2013;39(07):762-771

37 Fanin M, Nascimbeni AC, Angelini C. Gender difference in limbgirdle muscular dystrophy: a muscle fiber morphometric study in 101 patients. Clin Neuropathol 2014;33(03):179-185

38 Groen EJ, Charlton R, Barresi R, et al. Analysis of the UK diagnostic strategy for limb girdle muscular dystrophy 2A. Brain 2007;130 (Pt 12):3237-3249

39 Dirik E, Aydin A, Kurul S, Sahin B. Limb girdle muscular dystrophy type 2A presenting with cardiac arrest. Pediatr Neurol 2001;24 (03):235-237

40 Piluso G, Politano L, Aurino S, et al. Extensive scanning of the calpain-3 gene broadens the spectrum of LGMD2A phenotypes. J Med Genet 2005;42(09):686-693

41 de Paula F, Vainzof M, Passos-Bueno MR, et al. Clinical variability in calpainopathy: what makes the difference? Eur J Hum Genet 2002;10(12):825-832 